MATHEMATICS OF COMPUTATION

Volume 74, Number 249, Pages 103-122

S 0025-5718(04)01655-2

Article electronically published on April 22, 2004

\title{
FIRST AND SECOND ORDER ERROR ESTIMATES FOR THE UPWIND SOURCE AT INTERFACE METHOD
}

\author{
THEODOROS KATSAOUNIS AND CHIARA SIMEONI
}

\begin{abstract}
The Upwind Source at Interface (U.S.I.) method for hyperbolic conservation laws with source term introduced by Perthame and Simeoni is essentially first order accurate. Under appropriate hypotheses of consistency on the finite volume discretization of the source term, we prove $L^{p}$-error estimates, $1 \leq p<+\infty$, in the case of a uniform spatial mesh, for which an optimal result can be obtained. We thus conclude that the same convergence rates hold as for the corresponding homogeneous problem. To improve the numerical accuracy, we develop two different approaches of dealing with the source term and we discuss the question of deriving second order error estimates. $\mathrm{Nu}$ merical evidence shows that those techniques produce high resolution schemes compatible with the U.S.I. method.
\end{abstract}

\section{INTRODUCTION}

We consider the initial value problem for a transport equation with nonlinear source term, in one space dimension,

$$
\begin{aligned}
& \partial_{t} u+\partial_{x} u=B(x, u), \quad t \in \mathbb{R}_{+}, x \in \mathbb{R}, \\
& u(0, x)=u_{0}(x) \in L^{p}(\mathbb{R}) \cap L^{\infty}(\mathbb{R}), 1 \leq p<+\infty,
\end{aligned}
$$

with $u(t, x) \in \mathbb{R}$ and the analytical source operator is defined as

$$
B(x, u)=z^{\prime}(x) b(u), \quad z^{\prime} \in L^{p}(\mathbb{R}), b \in C^{1}(\mathbb{R}) .
$$

The system (1.1) - (1.3) is a simple model of scalar conservation laws with "geometrical" source term and it is motivated by the shallow water equations.

The entropy inequalities associated with (1.1) are described by the equation

$$
\partial_{t} S(u)+\partial_{x} S(u)+S^{\prime}(u) B(x, u) \leq 0,
$$

for any convex real-valued entropy function $S$ (refer to 7 , for instance).

Under stronger assumptions on the source term (1.3), Kružkov 17 proved existence and uniqueness of the entropy solution to the problem (1.1)-(1.2) in $L^{\infty}\left([0, T) ; L^{p}(\mathbb{R})\right)$, for all $T \in \mathbb{R}_{+}$.

Several results concerning the convergence analysis of numerical approximations for scalar conservation laws are inspired by this fundamental theory (see references

Received by the editor March 20, 2003 and, in revised form, July 8, 2003.

2000 Mathematics Subject Classification. Primary 65N15, 35L65, 74S10.

Key words and phrases. Scalar conservation laws, source terms, finite volume schemes, upwind interfacial methods, consistency, error estimates.

This work is partially supported by HYKE European programme HPRN-CT-2002-00282 (http://www.hyke.org). The authors would like to thank Professor B. Perthame for his valuable help and Professor Ch. Makridakis for helpful discussions. 
in [29] and [3], 11], [19], [26], 27], for instance). Another approach to the global existence of weak solutions for hyperbolic systems of balance laws is presented in [8], which is also referred to [12] with some extensions. In the particular case of singular source terms, a uniqueness result has recently been proved in [33].

In this paper, we consider finite volume discretizations of (1.1)-1.2) and we estimate the error induced by these discretizations. In particular, the source term $B(x, u)$ in (1.3) is approximated by a discrete operator $B^{h}$ of finite volume type. The solution $u$ and the function $z$ are approximated by piecewise constants in each grid cell, while the differential part of (1.1) is not discretized and it is kept in continuous form (2.4). The discrete source operator $B^{h}$ satisfies certain consistency properties (2.7)-(2.9), which are crucial for the convergence analysis.

We first consider piecewise constant approximations of $u$ and $z$ in each grid cell and we prove in Theorem 2.3 that, under certain regularity assumptions on $u$ and $z$, the resulting scheme is first order accurate.

A main theme in this work is to construct second order approximations of the source term. It turns out that second order accuracy is not obtained by a straightforward extension of the standard piecewise linear reconstruction technique to the source term. Our approach is based on constructing approximations with piecewise linear reconstructions in each grid cell for $u$ as well as for $z$ and the second order accuracy is achieved if an extra central term is added to the discrete source operator $B^{h}$ in (2.13). Additional hypotheses are required on the slope limiters used for the piecewise linear reconstructions; see Section 4. We prove in Theorem 2.4 that, under these hypotheses, our scheme satisfies a second error estimate.

We also consider an alternative way to obtain second order accuracy. In this case, piecewise linear approximations are used for the solution $u$, while piecewise constant approximations are used for $z$ in each grid cell; see (2.14). However, the discrete source operator $B^{h}$ has to satisfy a slightly stronger consistency condition (2.15) in order to achieve second order accuracy; see Theorem 2.5.

The rest of the paper is organized as follows. In Section 2, we introduce the necessary preliminary material and we state our convergence results. In Section 3 and Section 4, we derive the first and second order error estimates, respectively. We conclude with some final remarks in Section 5.

\section{NotATION AND PRELIMINARIES}

We set up a uniform mesh on $\mathbb{R}$, whose vertices are $x_{i}, i \in \mathbb{Z}$, and we denote by $C_{i}=\left[x_{i-\frac{1}{2}}, x_{i+\frac{1}{2}}\right)$ the control volume (cell), where $x_{i+\frac{1}{2}}=\frac{x_{i}+x_{i+1}}{2}$ are the cell interfaces and the characteristic space-step is $h=\operatorname{length}\left(C_{i}\right), \forall i \in \mathbb{Z}$ (see Figure 1). Then, we construct a piecewise constant approximation $z^{h}$ of the function $z$ on the mesh by

$$
z^{h}(x)=\sum_{i \in \mathbb{Z}} z_{i} \mathbb{1}_{C_{i}}(x), \quad z_{i}=\frac{1}{h} \int_{C_{i}} z(x) d x,
$$

where $\mathbb{1}_{C_{i}}$ denotes the characteristic function of the cell $C_{i}$.

We also introduce a piecewise constant approximation $u^{h}$ of the analytical solution $u$ of (1.1) $-(1.2)$, given by

$$
u^{h}(t, x)=\sum_{i \in \mathbb{Z}} u_{i}(t) \mathbb{1}_{C_{i}}(x), \quad u_{i}(t)=\frac{1}{h} \int_{C_{i}} u(t, x) d x .
$$




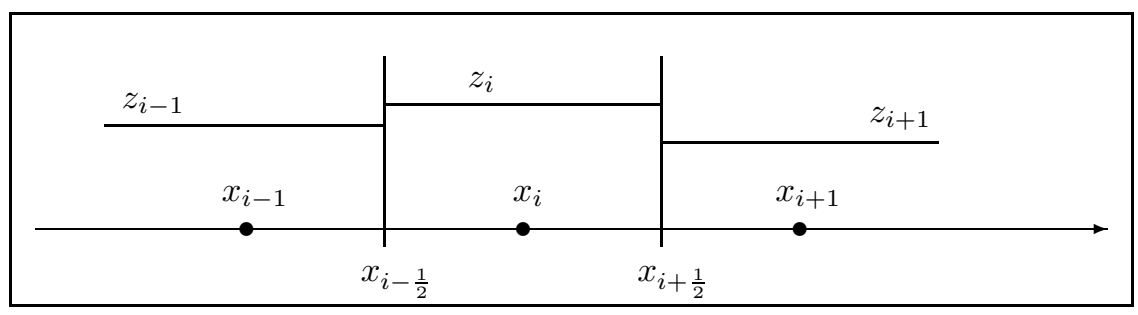

FiguRE 1. Constant reconstruction on the spatial mesh

In the above framework, the numerical solution obtained from a finite volume scheme applied to (1.1)-(1.2) is a discrete function $v^{h}$, whose cell-averages

$$
v_{i}(t)=\frac{1}{h} \int_{C_{i}} v^{h}(t, x) d x, \quad i \in \mathbb{Z},
$$

are interpreted as approximations of the cell-averages of the analytical solution, i.e. $v_{i}(t) \approx u_{i}(t), i \in \mathbb{Z}$. The general semi-discrete scheme for (1.1) reads

$$
\partial_{t} v^{h}+\partial_{x} v^{h}=B^{h}\left(x, v^{h}\right),
$$

with initial data corresponding to the approximate initial condition

$$
v_{0}^{h}(x)=\sum_{i \in \mathbb{Z}} v_{i}(0) \mathbb{1}_{C_{i}}(x), \quad v_{i}(0)=\frac{1}{h} \int_{C_{i}} u_{0}(x) d x .
$$

The source term in (2.4) is discretized as (see [29])

$$
B^{h}\left(x, v^{h}\right)=\sum_{i \in \mathbb{Z}} \frac{1}{h}\left[\mathcal{B}^{+}\left(v_{i-1}, v_{i}, \Delta z_{i-\frac{1}{2}}\right)+\mathcal{B}^{-}\left(v_{i}, v_{i+1}, \Delta z_{i+\frac{1}{2}}\right)\right] \mathbb{1}_{C_{i}},
$$

where we set $\Delta z_{i+\frac{1}{2}}=z_{i+1}-z_{i}$ (here and in the sequel, we drop time and space dependence in the formulas for simplicity). We assume the following consistency properties for the discrete source operator (2.6), with respect to (1.3), which are fundamental for the convergence analysis,

$$
\begin{aligned}
\mathcal{B}^{ \pm} \in C^{2}, \quad \mathcal{B}^{ \pm}(u, v, 0)= & 0, \quad \frac{\partial \mathcal{B}^{ \pm}}{\partial u}(u, v, 0)=\frac{\partial \mathcal{B}^{ \pm}}{\partial v}(u, v, 0)=0, \\
& \lim _{\lambda \rightarrow 0} \frac{\mathcal{B}^{+}(u, u, \lambda)+\mathcal{B}^{-}(u, u, \lambda)}{\lambda}=b(u) .
\end{aligned}
$$

The last limit holds uniformly in $u$, in particular we assume that

$$
\left|\frac{\mathcal{B}^{+}(u, u, \lambda)+\mathcal{B}^{-}(u, u, \lambda)}{\lambda}-b(u)\right| \leq K_{B} \lambda,
$$

where $K_{B}$ is a constant (independent of $u$ ). Moreover, we denote by $L_{B}$ any Lipschitz constant associated to the continuous or discrete source operator.

Remark 2.1. We note that the definition of consistency (2.8) for the source term refers to the interpretation of the upwind interfacial discretization (2.6) in the sense of finite volume methods. 


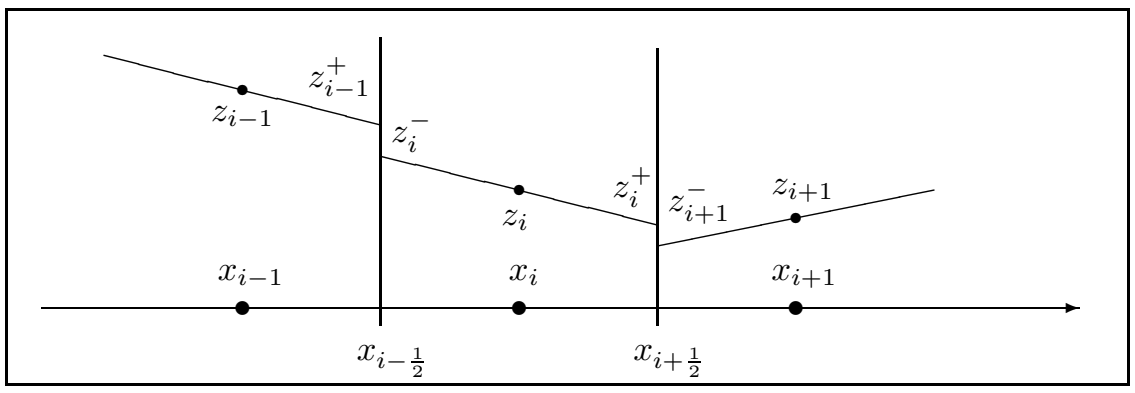

FiguRE 2. Linear reconstruction on the spatial mesh

2.1. Second order extension of the Upwind Source at Interface method. In order to obtain second order extensions of the discrete solver (2.4)-(2.5), we apply a slope limiter technique to the numerical functions: the basic idea is to replace the piecewise constant reconstruction of the approximate solution by more accurate reconstructions, namely piecewise linear (see [10] and 20]).

We associate to the numerical solution some coefficients, defined as second order interpolation of the discrete unknowns (2.3), i.e.,

$$
\bar{v}_{i}(t, x)=v_{i}(t)+\left(x-x_{i}\right) v_{i}^{\prime}, \quad i \in \mathbb{Z}, x \in C_{i},
$$

where $v_{i}^{\prime}$ indicates a generic numerical derivative, computed by means of an appropriate slope limiter (see Section 4). Departing from (2.1) and (2.2), the function $z$ and the analytical solution $u$ can also be represented in terms of piecewise linear approximations on the spatial mesh, with coefficients

$$
\begin{gathered}
\bar{z}_{i}(x)=z_{i}+\left(x-x_{i}\right) z_{i}^{\prime}, \quad i \in \mathbb{Z}, x \in C_{i}, \\
\bar{u}_{i}(t, x)=u_{i}(t)+\left(x-x_{i}\right) u_{i}^{\prime}, \quad i \in \mathbb{Z}, x \in C_{i} .
\end{gathered}
$$

At the cell interfaces, the values of the numerical functions are given by

$$
\begin{aligned}
v_{i}^{-} & =\bar{v}_{i}\left(x_{i-\frac{1}{2}}\right)=v_{i}-\frac{h}{2} v_{i}^{\prime}, & v_{i}^{+} & =\bar{v}_{i}\left(x_{i+\frac{1}{2}}\right)=v_{i}+\frac{h}{2} v_{i}^{\prime}, \\
z_{i}^{-} & =\bar{z}_{i}\left(x_{i-\frac{1}{2}}\right)=z_{i}-\frac{h}{2} z_{i}^{\prime}, & z_{i}^{+} & =\bar{z}_{i}\left(x_{i+\frac{1}{2}}\right)=z_{i}+\frac{h}{2} z_{i}^{\prime},
\end{aligned}
$$

so that $\Delta z_{i+\frac{1}{2}}=z_{i+1}^{-}-z_{i}^{+}$, for example (see Figure 2).

Therefore, it is natural to perform a discretization of the source term (1.3) by using the interfacial values (2.11) and (2.12), that is

$$
\begin{aligned}
B^{h}\left(x, v^{h}\right) & =\sum_{i \in \mathbb{Z}} \frac{1}{h}\left[\mathcal{B}^{+}\left(v_{i-1}^{+}, v_{i}^{-}, \Delta z_{i-\frac{1}{2}}\right)+\mathcal{B}^{-}\left(v_{i}^{+}, v_{i+1}^{-}, \Delta z_{i+\frac{1}{2}}\right)\right] \mathbb{1}_{C_{i}} \\
& +\sum_{i \in \mathbb{Z}} z_{i}^{\prime} b\left(v_{i}\right) \mathbb{1}_{C_{i}},
\end{aligned}
$$

with an additional term in comparison to the discrete source operator (2.6) , which depends on the cell-averages and is necessary to achieve second order estimates (refer to Section 5 for further details).

An alternative approach to formulating second order extensions of the U.S.I. method in form (2.6) is based on improving the consistency properties of the numerical source operator. We consider a piecewise constant approximation (2.1) 
of the function $z$ on the mesh and piecewise linear reconstructions (2.10) of the numerical solution to define the upwind interfacial discretization

$$
B^{h}\left(x, v^{h}\right)=\sum_{i \in \mathbb{Z}} \frac{1}{h}\left[\mathcal{B}^{+}\left(v_{i-1}^{+}, v_{i}^{-}, \Delta z_{i-\frac{1}{2}}\right)+\mathcal{B}^{-}\left(v_{i}^{+}, v_{i+1}^{-}, \Delta z_{i+\frac{1}{2}}\right)\right] \mathbb{1}_{C_{i}},
$$

where the numerical functions are computed on the interfacial values (2.11) and $\Delta z_{i+\frac{1}{2}}=z_{i+1}-z_{i}$. This is suggested by the particular form of source term (1.3), given by the product of functions which exhibit different orders of derivative. To obtain second order accuracy, we need to assume that (2.8) holds, along with the second order hypothesis of consistency

$$
\left|\frac{\mathcal{B}^{+}(u, u, \lambda)+\mathcal{B}^{-}(u, u, \lambda)}{\lambda}-b(u)\right| \leq K_{B} \lambda^{2} .
$$

Remark 2.2. In effect, the discretizations (2.13) and (2.14) are strictly related, as formally verified by means of standard asymptotic expansions on the numerical functions and simple algebraic calculations with the differences of discrete values (2.1) or (2.12). We also note that many of the second order schemes proposed in the literature do not include the additional term (2.13), but it is probably recovered implicitly in the formulation (see [1], 223] and [28], for instance).

2.2. Convergence and error estimates. To deal with the question of deriving error estimates for the numerical approximation (2.4) $-(2.5)$ to the problem (1.1)(1.2), we introduce the error function

$$
e(t, x)=u(t, x)-v^{h}(t, x),
$$

which satisfies the equation

$$
\begin{aligned}
\partial_{t} e+\partial_{x} e & =B(x, u)-B^{h}\left(x, v^{h}\right) \\
& =\left[B(x, u)-B^{h}\left(x, u^{h}\right)\right]+\left[B^{h}\left(x, u^{h}\right)-B^{h}\left(x, v^{h}\right)\right] \\
& :=\mathcal{C}\left(u ; u^{h}\right)+\mathcal{S}\left(u^{h} ; v^{h}\right) .
\end{aligned}
$$

From (2.2) and (2.3), we obtain the usual expression for the cell-averages,

$$
e_{i}(t)=\frac{1}{h} \int_{C_{i}} e(t, x) d x=u_{i}(t)-v_{i}(t), \quad i \in \mathbb{Z} .
$$

The operators $\mathcal{C}\left(u ; u^{h}\right)$ and $\mathcal{S}\left(u^{h} ; v^{h}\right)$ in formula (2.17) denote the consistency and stability error terms, respectively. Now we state our main convergence results.

Theorem 2.3. We assume $z \in W^{2, p}, 1 \leq p<+\infty$, and we consider the discrete source operator (2.6) in (2.17). Then, for all $t \in \mathbb{R}_{+}$, the error function (2.16) verifies the first order estimate

$$
\|e(t)\|_{L^{p}} \leq C(t)\left(\left\|e_{0}\right\|_{L^{p}}+h\|z\|_{W^{2, p}}+h \int_{0}^{t} \exp \{-C s\}\|u(s)\|_{W^{1, p}} d s\right),
$$

where $C(t)$ is a constant independent of $h$.

The convergence properties of second order schemes are notably affected by the technique used to construct piecewise linear approximations of the numerical functions, namely the choice of the slope limiter [15], [25], [35]. Without some appropriate hypotheses on the coefficients of such approximations, the proof of the consistency estimate given in Section 4.2 fails and numerical evidence shows that 
the discretization (2.13) loses second order accuracy (refer to Section 5 for details). The following results extend the one which is established in Theorem [2.3] to the discretizations (2.13) and (2.14).

Theorem 2.4. We assume $z \in W^{3, p}, 1 \leq p<+\infty$, and we consider the discrete source operator (2.13), with numerical derivatives computed in the restricted class of slope limiters introduced in Section 4. Then, for all $t \in \mathbb{R}_{+}$, the error function (2.16) verifies the second order estimate

$$
\|e(t)\|_{L^{p}} \leq C(t)\left(\left\|e_{0}\right\|_{L^{p}}+h^{2}\|z\|_{W^{3, p}}+h^{2} \int_{0}^{t} \exp \{-C s\}\|u(s)\|_{W^{2, p}} d s\right),
$$

where $C(t)$ is a constant independent of $h$.

Theorem 2.5. We assume $z \in W^{3, p}, 1 \leq p<+\infty$, and we consider the discrete source operator (2.14) in (2.17), with the consistency property (2.15). Then, for all $t \in \mathbb{R}_{+}$, the error function (2.16) verifies the second order estimate

$$
\|e(t)\|_{L^{p}} \leq C(t)\left(\left\|e_{0}\right\|_{L^{p}}+h^{2}\|z\|_{W^{3, p}}+h^{2} \int_{0}^{t} \exp \{-C s\}\|u(s)\|_{W^{2, p}} d s\right),
$$

where $C(t)$ is a constant independent of $h$.

The suitable convergence of initial data in (2.19), 2.20) and (2.21), as the mesh size tends to zero, is guaranteed by the first and second order convergence of piecewise constant approximations. Indeed, because of definitions (2.2) and (2.5), we have $v_{0}^{h}=u_{0}^{h}$ and we deduce from (2.16) that $e_{0}(x)=u_{0}(x)-u_{0}^{h}(x), x \in \mathbb{R}$. Besides, the following statements are classical and are not difficult to prove:

$$
\begin{aligned}
& \left\|e_{0}\right\|_{L^{p}} \leq C h \quad \text { if } \quad u_{0} \in W^{1, p}, 1 \leq p<+\infty \\
& \left\|e_{0}\right\|_{L^{p}} \leq C h^{2} \quad \text { if } \quad u_{0} \in W^{2, p}, 1 \leq p<+\infty
\end{aligned}
$$

for some constant $C$ independent of $h$.

Remark 2.6. The arguments presented in this paper do not apply directly to nonlinear scalar conservation laws with a source term, to derive complete error estimates for the U.S.I. method.

\section{ERROR ESTIMATES FOR FIRST ORDER SCHEMES}

Before giving details of the estimates, we introduce some relations on the discrete differences of numerical functions that we will frequently use later on the proofs. We consider a function $w \in C^{1}$ whose cell-averages on the spatial mesh are given by $w_{i}=\frac{1}{h} \int_{C_{i}} w(x) d x, i \in \mathbb{Z}$. By performing appropriate expansions, we obtain

$$
\begin{gathered}
w_{i+1}-w_{i}=\int_{C_{i}} w^{\prime}(\xi(x)) d x=h w^{\prime}\left(x_{i}\right)+\int_{C_{i}} w^{\prime \prime}(\eta(x))\left(x-x_{i}\right) d x \\
w_{i+1}-2 w_{i}+w_{i-1}=h \int_{C_{i}} w^{\prime \prime}(\vartheta(x)) d x
\end{gathered}
$$

for some $\xi(x), \eta(x), \vartheta(x) \in C_{i}$. We also recall the classical Taylor formula, in the particular form with an integral expression for the remainder,

$$
w(x)=\sum_{k=0}^{n} \frac{1}{k !} w^{k}\left(x_{i}\right)\left(x-x_{i}\right)^{k}+\frac{1}{n !} \int_{x_{i}}^{x}(x-s)^{n} w^{n+1}(s) d s .
$$


3.1. Stability estimate. We begin by estimating the stability term $\mathcal{S}\left(u^{h} ; v^{h}\right)$ in equation (2.17).

Lemma 3.1. For the assumptions of Theorem 2.3, together with (2.7), there exists a constant $C:=C\left(L_{B},\left\|z^{\prime}\right\|_{L^{\infty}}\right)$, independent of $h$, such that

$$
\left.\left|\int_{\mathbb{R}} \mathcal{S}\left(u^{h} ; v^{h}\right)\right| e\right|^{p-1} \operatorname{sgn}(e) d x \mid \leq C\|e\|_{L^{p}}^{p} .
$$

Proof. From (2.17), according to (2.2) and (2.6), we deduce that

$$
\begin{array}{rl}
\int_{\mathbb{R}} & \mathcal{S}\left(u^{h} ; v^{h}\right)|e|^{p-1} \operatorname{sgn}(e) d x=\int_{\mathbb{R}}\left[B^{h}\left(x, u^{h}\right)-B^{h}\left(x, v^{h}\right)\right]|e|^{p-1} \operatorname{sgn}(e) d x \\
=\int_{\mathbb{R}}\left\{\sum_{i \in \mathbb{Z}} \frac{1}{h}\left[\mathcal{B}^{+}\left(u_{i-1}, u_{i}, \Delta z_{i-\frac{1}{2}}\right)+\mathcal{B}^{-}\left(u_{i}, u_{i+1}, \Delta z_{i+\frac{1}{2}}\right)\right] \mathbb{1}_{C_{i}}\right. \\
\left.\quad-\sum_{i \in \mathbb{Z}} \frac{1}{h}\left[\mathcal{B}^{+}\left(v_{i-1}, v_{i}, \Delta z_{i-\frac{1}{2}}\right)+\mathcal{B}^{-}\left(v_{i}, v_{i+1}, \Delta z_{i+\frac{1}{2}}\right)\right] \mathbb{1}_{C_{i}}\right\}|e|^{p-1} \operatorname{sgn}(e) d x \\
=\sum_{i \in \mathbb{Z}}\left[\mathcal{B}^{+}\left(u_{i-1}, u_{i}, \Delta z_{i-\frac{1}{2}}\right)-\mathcal{B}^{+}\left(v_{i-1}, v_{i}, \Delta z_{i-\frac{1}{2}}\right)\right] e_{i}^{p-1} \\
\quad+\sum_{i \in \mathbb{Z}}\left[\mathcal{B}^{-}\left(u_{i}, u_{i+1}, \Delta z_{i+\frac{1}{2}}\right)-\mathcal{B}^{-}\left(v_{i}, v_{i+1}, \Delta z_{i+\frac{1}{2}}\right)\right] e_{i}^{p-1} \\
=\sum_{i \in \mathbb{Z}}\left[\mathcal{B}^{+}\left(u_{i}, u_{i+1}, \Delta z_{i+\frac{1}{2}}\right)-\mathcal{B}^{+}\left(v_{i}, v_{i+1}, \Delta z_{i+\frac{1}{2}}\right)\right] e_{i+1}^{p-1} \\
\quad+\sum_{i \in \mathbb{Z}}\left[\mathcal{B}^{-}\left(u_{i}, u_{i+1}, \Delta z_{i+\frac{1}{2}}\right)-\mathcal{B}^{-}\left(v_{i}, v_{i+1}, \Delta z_{i+\frac{1}{2}}\right)\right] e_{i}^{p-1}:=S_{1}+S_{2},
\end{array}
$$

where we have set $e_{i}^{p-1}=\frac{1}{h} \int_{C_{i}}|e|^{p-1} \operatorname{sgn}(e) d x$. We estimate the terms $S_{1}$ and $S_{2}$ separately. In view of properties (2.7), we have

$$
\begin{aligned}
S_{1} & =\sum_{i \in \mathbb{Z}}\left[\mathcal{B}^{+}\left(u_{i}, u_{i+1}, \Delta z_{i+\frac{1}{2}}\right)-\mathcal{B}^{+}\left(v_{i}, u_{i+1}, \Delta z_{i+\frac{1}{2}}\right)\right] e_{i+1}^{p-1} \\
& +\sum_{i \in \mathbb{Z}}\left[\mathcal{B}^{+}\left(v_{i}, u_{i+1}, \Delta z_{i+\frac{1}{2}}\right)-\mathcal{B}^{+}\left(v_{i}, v_{i+1}, \Delta z_{i+\frac{1}{2}}\right)\right] e_{i+1}^{p-1} \\
& =\sum_{i \in \mathbb{Z}}\left(\int_{v_{i}}^{u_{i}}\left[\frac{\partial \mathcal{B}^{+}}{\partial u}\left(u, u_{i+1}, \Delta z_{i+\frac{1}{2}}\right)-\frac{\partial \mathcal{B}^{+}}{\partial u}\left(u, u_{i+1}, 0\right)\right] d u\right) e_{i+1}^{p-1} \\
& +\sum_{i \in \mathbb{Z}}\left(\int_{v_{i+1}}^{u_{i+1}}\left[\frac{\partial \mathcal{B}^{+}}{\partial v}\left(v_{i}, v, \Delta z_{i+\frac{1}{2}}\right)-\frac{\partial \mathcal{B}^{+}}{\partial v}\left(v_{i}, v, 0\right)\right] d v\right) e_{i+1}^{p-1}
\end{aligned}
$$

and we conclude that

$$
S_{1} \leq L_{B} \sum_{i \in \mathbb{Z}}\left|\Delta z_{i+\frac{1}{2}}\right|\left(\left|u_{i}-v_{i}\right|+\left|u_{i+1}-v_{i+1}\right|\right)\left|e_{i+1}^{p-1}\right| .
$$

We proceed similarly for $S_{2}$, also recalling (2.18), and we get

$$
\begin{aligned}
& S_{1} \leq L_{B} \sum_{i \in \mathbb{Z}}\left|\Delta z_{i+\frac{1}{2}}\right|\left(\left|e_{i}\right|+\left|e_{i+1}\right|\right)\left|e_{i+1}^{p-1}\right|, \\
& S_{2} \leq L_{B} \sum_{i \in \mathbb{Z}}\left|\Delta z_{i+\frac{1}{2}}\right|\left(\left|e_{i}\right|+\left|e_{i+1}\right|\right)\left|e_{i}^{p-1}\right| .
\end{aligned}
$$


By Hölder's inequality for $1 \leq p<+\infty$, we obtain $\left|e_{i}^{p-1}\right| \leq\left|e_{i}\right|^{p-1}$. This implies, after rearranging terms in (3.7)-(3.8) and using Young's inequality, with the immediate property $\left|e_{i}\right|^{p} \leq \frac{1}{h} \int_{C_{i}}|e|^{p} d x$, that

$$
\begin{aligned}
& \left.\left|\int_{\mathbb{R}} \mathcal{S}\left(u^{h} ; v^{h}\right)\right| e\right|^{p-1} \operatorname{sgn}(e) d x \mid \\
& \leq L_{B} \sum_{i \in \mathbb{Z}}\left|\Delta z_{i+\frac{1}{2}}\right|\left(\left|e_{i}\right|^{p}+\left|e_{i}\right|^{p-1}\left|e_{i+1}\right|+\left|e_{i}\right|\left|e_{i+1}\right|^{p-1}+\left|e_{i+1}\right|^{p}\right) \\
& \leq 2 L_{B} \sum_{i \in \mathbb{Z}} \frac{\left|\Delta z_{i+\frac{1}{2}}\right|}{h}\left(\int_{C_{i}}|e|^{p} d x+\int_{C_{i+1}}|e|^{p} d x\right) .
\end{aligned}
$$

In the case of (2.1), by applying 3.1), a direct estimate provides the first order approximation $\frac{\left|\Delta z_{i+\frac{1}{2}}\right|}{h} \leq\left\|z^{\prime}\right\|_{L^{\infty}}$. The proof of (3.4) is thus completed.

3.2. Consistency estimate. We turn our attention to the consistency error term $\mathcal{C}\left(u ; u^{h}\right)$ in (2.17), for which an optimal result in terms of the rate of convergence is obtained.

Lemma 3.2. For the assumptions of Theorem [2.3, together with (2.7)-(2.9), there exists a constant $C:=C\left(L_{B}, K_{B},\left\|z^{\prime}\right\|_{L^{\infty}}\right)$, independent of $h$, such that

$$
\left.\left|\int_{\mathbb{R}} \mathcal{C}\left(u ; u^{h}\right)\right| e\right|^{p-1} \operatorname{sgn}(e) d x \mid \leq C h\left(\|z\|_{W^{2, p}}+\|u\|_{W^{1, p}}\right)\|e\|_{L^{p}}^{p-1} .
$$

Proof. From 2.17), we have

$$
\int_{\mathbb{R}} \mathcal{C}\left(u ; u^{h}\right)|e|^{p-1} \operatorname{sgn}(e) d x=\int_{\mathbb{R}}\left[B(x, u)-B^{h}\left(x, u^{h}\right)\right]|e|^{p-1} \operatorname{sgn}(e) d x .
$$

Setting $\mathcal{B}=\mathcal{B}^{+}+\mathcal{B}^{-}$and $e_{i}^{p-1}=\frac{1}{h} \int_{C_{i}}|e|^{p-1} \operatorname{sgn}(e) d x$, we compute the integral of the discrete source operator as follows:

$$
\begin{aligned}
& \int_{\mathbb{R}} B^{h}\left(x, u^{h}\right)|e|^{p-1} \operatorname{sgn}(e) d x \\
= & \int_{\mathbb{R}}\left\{\sum_{i \in \mathbb{Z}} \frac{1}{h}\left[\mathcal{B}^{+}\left(u_{i-1}, u_{i}, \Delta z_{i-\frac{1}{2}}\right)+\mathcal{B}^{-}\left(u_{i}, u_{i+1}, \Delta z_{i+\frac{1}{2}}\right)\right] \mathbb{1}_{C_{i}}\right\}|e|^{p-1} \operatorname{sgn}(e) d x \\
= & \sum_{i \in \mathbb{Z}} \mathcal{B}\left(u_{i}, u_{i}, \Delta z_{i+\frac{1}{2}}\right) e_{i}^{p-1}+\sum_{i \in \mathbb{Z}}\left[\mathcal{B}\left(u_{i}, u_{i+1}, \Delta z_{i+\frac{1}{2}}\right)-\mathcal{B}\left(u_{i}, u_{i}, \Delta z_{i+\frac{1}{2}}\right)\right] e_{i}^{p-1} \\
+ & \sum_{i \in \mathbb{Z}}\left[\mathcal{B}^{+}\left(u_{i-1}, u_{i}, \Delta z_{i-\frac{1}{2}}\right)-\mathcal{B}^{+}\left(u_{i}, u_{i+1}, \Delta z_{i+\frac{1}{2}}\right)\right] e_{i}^{p-1}:=T_{1}+T_{2}+T_{3} .
\end{aligned}
$$

We estimate each term separately. For $T_{2}$, we use (2.7) to write

$$
\begin{aligned}
T_{2} & =\sum_{i \in \mathbb{Z}}\left(\int_{u_{i}}^{u_{i+1}}\left[\frac{\partial \mathcal{B}}{\partial v}\left(u_{i}, v, \Delta z_{i+\frac{1}{2}}\right)-\frac{\partial \mathcal{B}}{\partial v}\left(u_{i}, v, 0\right)\right] d v\right) e_{i}^{p-1} \\
& \leq L_{B} \sum_{i \in \mathbb{Z}}\left|\Delta z_{i+\frac{1}{2}}\right|\left|u_{i+1}-u_{i}\right|\left|e_{i}^{p-1}\right| .
\end{aligned}
$$

Then we consider (3.1) applied to (2.1) and (2.2) for concluding that

$$
T_{2} \leq L_{B}\left\|z^{\prime}\right\|_{L^{\infty}} \sum_{i \in \mathbb{Z}}\left(\int_{C_{i}}\left|u^{\prime}\right| d x\right)\left(\int_{C_{i}}|e|^{p-1} d x\right)
$$


Similarly for the last term $T_{3}$, we proceed as in (3.5) and (3.6) to get

$$
\begin{aligned}
T_{3}= & \sum_{i \in \mathbb{Z}}\left[\mathcal{B}^{+}\left(u_{i-1}, u_{i}, \Delta z_{i-\frac{1}{2}}\right)-\mathcal{B}^{+}\left(u_{i}, u_{i}, \Delta z_{i-\frac{1}{2}}\right)\right] e_{i}^{p-1} \\
& +\sum_{i \in \mathbb{Z}}\left[\mathcal{B}^{+}\left(u_{i}, u_{i}, \Delta z_{i-\frac{1}{2}}\right)-\mathcal{B}^{+}\left(u_{i}, u_{i}, \Delta z_{i+\frac{1}{2}}\right)\right] e_{i}^{p-1} \\
& +\sum_{i \in \mathbb{Z}}\left[\mathcal{B}^{+}\left(u_{i}, u_{i}, \Delta z_{i+\frac{1}{2}}\right)-\mathcal{B}^{+}\left(u_{i}, u_{i+1}, \Delta z_{i+\frac{1}{2}}\right)\right] e_{i}^{p-1} \\
\leq & L_{B} \sum_{i \in \mathbb{Z}}\left(\left|\Delta z_{i-\frac{1}{2}}\right|\left|u_{i}-u_{i-1}\right|+\left|\Delta z_{i+\frac{1}{2}}-\Delta z_{i-\frac{1}{2}}\right|+\left|\Delta z_{i+\frac{1}{2}}\right|\left|u_{i+1}-u_{i}\right|\right)\left|e_{i}^{p-1}\right|
\end{aligned}
$$

from which, by means of (3.1) and (3.2), we conclude that

$$
\begin{aligned}
T_{3} & \leq 2 L_{B}\left\|z^{\prime}\right\|_{L^{\infty}} \sum_{i \in \mathbb{Z}}\left(\int_{C_{i}}\left|u^{\prime}\right| d x\right)\left(\int_{C_{i}}|e|^{p-1} d x\right) \\
& +L_{B} \sum_{i \in \mathbb{Z}}\left(\int_{C_{i}}\left|z^{\prime \prime}\right| d x\right)\left(\int_{C_{i}}|e|^{p-1} d x\right) .
\end{aligned}
$$

Concerning the principal term $T_{1}$, it can be further decomposed into three parts

$$
\begin{aligned}
T_{1} & =\sum_{i \in \mathbb{Z}} \frac{\mathcal{B}\left(u_{i}, u_{i}, \Delta z_{i+\frac{1}{2}}\right)}{\Delta z_{i+\frac{1}{2}}}\left[\frac{\Delta z_{i+\frac{1}{2}}}{h}-z^{\prime}\left(x_{i}\right)\right] h e_{i}^{p-1} \\
& +\sum_{i \in \mathbb{Z}}\left[\frac{\mathcal{B}\left(u_{i}, u_{i}, \Delta z_{i+\frac{1}{2}}\right)}{\Delta z_{i+\frac{1}{2}}}-b\left(u_{i}\right)\right] z^{\prime}\left(x_{i}\right) h e_{i}^{p-1} \\
& +\sum_{i \in \mathbb{Z}} z^{\prime}\left(x_{i}\right) b\left(u_{i}\right) h e_{i}^{p-1}:=T_{1}^{1}+T_{1}^{2}+T_{1}^{3} .
\end{aligned}
$$

We give details for each part. From (3.1) and (2.7), we deduce that

$$
\begin{aligned}
T_{1}^{1} & \leq \sum_{i \in \mathbb{Z}}\left|\frac{\mathcal{B}\left(u_{i}, u_{i}, \Delta z_{i+\frac{1}{2}}\right)-\mathcal{B}\left(u_{i}, u_{i}, 0\right)}{\Delta z_{i+\frac{1}{2}}}\right|\left|\frac{\Delta z_{i+\frac{1}{2}}}{h}-z^{\prime}\left(x_{i}\right)\right| h\left|e_{i}^{p-1}\right| \\
& \leq L_{B} \sum_{i \in \mathbb{Z}}\left(\int_{C_{i}}\left|z^{\prime \prime}\right| d x\right)\left(\int_{C_{i}}|e|^{p-1} d x\right) .
\end{aligned}
$$

Because of the consistency property (2.9) and (3.1) applied to (2.1), we have

$$
T_{1}^{2} \leq K_{B}\left\|z^{\prime}\right\|_{L^{\infty}} \sum_{i \in \mathbb{Z}}\left(\int_{C_{i}}\left|z^{\prime}\right| d x\right)\left(\int_{C_{i}}|e|^{p-1} d x\right) .
$$

Finally, the third term in (3.15) is equivalent to the integral of the analytical source operator in (3.11). Indeed, by means of standard Taylor expansions, we obtain for (2.2) the midpoint formula as

$$
u_{i}=u\left(x_{i}\right)+R_{i}, \quad R_{i}=\frac{1}{h} \int_{C_{i}} u^{\prime}(\xi(x))\left(x-x_{i}\right) d x,
$$

for some $\xi(x) \in C_{i}$, and the regularity assumed for (1.3) guarantees that

$$
b\left(u_{i}\right)=b\left(u\left(x_{i}\right)\right)+b^{\prime}\left(\nu_{i}\right) R_{i}, \quad\left|b^{\prime}\left(\nu_{i}\right)\right| \leq L_{B}, \quad \forall i \in \mathbb{Z} .
$$


We thus write

$$
T_{1}^{3}=\sum_{i \in \mathbb{Z}} z^{\prime}\left(x_{i}\right) b\left(u\left(x_{i}\right)\right) h e_{i}^{p-1}+R_{1},
$$

where the remainder satisfies the estimate

$$
R_{1} \leq L_{B}\left\|z^{\prime}\right\|_{L^{\infty}} \sum_{i \in \mathbb{Z}}\left(\int_{C_{i}}\left|u^{\prime}\right| d x\right)\left(\int_{C_{i}}|e|^{p-1} d x\right) .
$$

The Taylor formula (3.3), applied to the source term (1.3), yields

$$
\int_{\mathbb{R}} B(x, u)|e|^{p-1} \operatorname{sgn}(e) d x=\sum_{i \in \mathbb{Z}} \int_{C_{i}} z^{\prime}\left(x_{i}\right) b\left(u\left(x_{i}\right)\right)|e|^{p-1} \operatorname{sgn}(e) d x+R_{2}
$$

and we readily check that the remainder satisfies

$$
\begin{aligned}
R_{2} & \leq L_{B} \sum_{i \in \mathbb{Z}}\left(\int_{C_{i}}\left|z^{\prime \prime}\right| d x\right)\left(\int_{C_{i}}|e|^{p-1} d x\right) \\
& +L_{B}\left\|z^{\prime}\right\|_{L^{\infty}} \sum_{i \in \mathbb{Z}}\left(\int_{C_{i}}\left|u^{\prime}\right| d x\right)\left(\int_{C_{i}}|e|^{p-1} d x\right) .
\end{aligned}
$$

Therefore, from (3.18) we conclude that

$$
T_{1}^{3}=\int_{\mathbb{R}} B(x, u)|e|^{p-1} \operatorname{sgn}(e) d x+R_{1}-R_{2},
$$

with the corresponding estimates (3.19) and (3.20). We combine (3.13), (3.14), (3.16), (3.17) and (3.21), we apply the discrete Hölder inequality for $1 \leq p<+\infty$ and then we deduce (3.10).

3.3. Proof of Theorem 2.3. We multiply equation (2.17) by $|e|^{p-1} \operatorname{sgn}(e)$ and we integrate to get

$$
\begin{aligned}
& \int_{\mathbb{R}}\left(\partial_{t} e+\partial_{x} e\right)|e|^{p-1} \operatorname{sgn}(e) d x \\
& \quad=\int_{\mathbb{R}} \mathcal{C}\left(u ; u^{h}\right)|e|^{p-1} \operatorname{sgn}(e) d x+\int_{\mathbb{R}} \mathcal{S}\left(u^{h} ; v^{h}\right)|e|^{p-1} \operatorname{sgn}(e) d x .
\end{aligned}
$$

An integration by parts in (3.22) shows that $\int_{\mathbb{R}} \partial_{x} e|e|^{p-1} \operatorname{sgn}(e) d x=0$; therefore we deduce from (3.4) and (3.10) that

$$
\frac{1}{p} \partial_{t}\|e(t)\|_{L^{p}}^{p} \leq C\|e(t)\|_{L^{p}}^{p}+C h\left(\|z\|_{W^{2, p}}+\|u(t)\|_{W^{1, p}}\right)\|e(t)\|_{L^{p}}^{p-1} .
$$

Let $t^{*} \in \mathbb{R}_{+}$be such that $\left\|e\left(t^{*}\right)\right\|_{L^{p}}=\max _{t \in \mathbb{R}_{+}}\|e(t)\|_{L^{p}}$. After integrating with respect to time in (3.23), we rearrange the estimate as

$$
\begin{aligned}
\left\|e\left(t^{*}\right)\right\|_{L^{p}}^{p} & \leq\left\|e_{0}\right\|_{L^{p}}\left\|e\left(t^{*}\right)\right\|_{L^{p}}^{p-1}+C p\left\|e\left(t^{*}\right)\right\|_{L^{p}}^{p-1} \int_{0}^{t^{*}}\|e(s)\|_{L^{p}} d s \\
& +C h p t^{*}\|z\|_{W^{2, p}}\left\|e\left(t^{*}\right)\right\|_{L^{p}}^{p-1}+C h p\left\|e\left(t^{*}\right)\right\|_{L^{p}}^{p-1} \int_{0}^{t^{*}}\|u(s)\|_{W^{1, p}} d s .
\end{aligned}
$$

Finally, a straightforward extension of Gronwall's inequality leads to the desired result in (2.19), where $C(t):=C\left(t ; p, L_{B}, K_{B},\left\|z^{\prime}\right\|_{L^{\infty}}\right)$ is some positive constant depending on time by the factor $\exp \{-C t\}$. 


\section{ERror ESTIMATES FOR SECOND ORDER SCHEMES}

The convergence properties of the approximations (2.13) and (2.14) are proved by means of analogous arguments as for (2.6) in Section 3 We first derive some preliminary estimates on the discrete differences of numerical functions.

For a function $w \in C^{2}$, with cell-averages $w_{i}=\frac{1}{h} \int_{C_{i}} w(x) d x, i \in \mathbb{Z}$, we construct piecewise linear approximations on the spatial mesh by using the coefficients

$$
\bar{w}_{i}(x)=w_{i}+\left(x-x_{i}\right) w_{i}^{\prime}, \quad i \in \mathbb{Z}, x \in C_{i},
$$

where the numerical derivatives are defined as appropriate interpolations of the discrete increments between neighboring cells, i.e.,

$$
w_{i}^{\prime}=\operatorname{lmtr}\left\{\frac{w_{i+1}-w_{i}}{h}, \frac{w_{i}-w_{i-1}}{h}\right\}, \quad i \in \mathbb{Z} .
$$

We consider a general representation of the slope limiter introduced in the above formula; namely if $M=\operatorname{lm} \operatorname{tr}\{\alpha, \beta\}$, then $M=\kappa \alpha+\lambda \beta$, with $\kappa, \lambda \in[0,1]$ and $\kappa+\lambda=1$ or $\kappa+\lambda=0$. In particular, we restrict our analysis to the special class of numerical operators in (4.2) which satisfy the condition $\kappa_{i}+\lambda_{i}=1, \forall i \in \mathbb{Z}$ (that excludes, for instance, the classical minmod limiter in the case of nonmonotonic functions). We also assume that the application (4.2), relating the cell-averages $w_{j}, j=i-1, i, i+1$, to the numerical derivative $w_{i}^{\prime}$ on the mesh cell, is Lipschitz continuous on its arguments, with constant $\frac{C}{h}$. Several examples of slope limiter which satisfy these properties have been formulated in the literature (see [13], [14], [24], 30] and 32], for instance).

We deduce from (4.2) and those definitions that

$$
w_{i}^{\prime}=\kappa_{i} \frac{w_{i+1}-w_{i}}{h}+\lambda_{i} \frac{w_{i}-w_{i-1}}{h}, \quad i \in \mathbb{Z} .
$$

The interfacial values of the reconstruction (4.1) are given by

$$
w_{i}^{-}=\bar{w}_{i}\left(x_{i-\frac{1}{2}}\right)=w_{i}-\frac{h}{2} w_{i}^{\prime}, \quad w_{i}^{+}=\bar{w}_{i}\left(x_{i+\frac{1}{2}}\right)=w_{i}+\frac{h}{2} w_{i}^{\prime}
$$

and we are interested in evaluating the jumps at the interfaces, i.e., $w_{i+1}^{-}-w_{i}^{+}$. Taking (4.3) and (4.4) into account, we have

$$
\begin{aligned}
w_{i+1}^{-}-w_{i}^{+} & =w_{i+1}-w_{i}-\frac{h}{2}\left(w_{i}^{\prime}+w_{i+1}^{\prime}\right) \\
& =\left(1-\frac{\kappa_{i}}{2}-\frac{\lambda_{i+1}}{2}\right) W_{i+\frac{1}{2}}-\frac{\lambda_{i}}{2} W_{i-\frac{1}{2}}-\frac{\kappa_{i+1}}{2} W_{i+\frac{3}{2}},
\end{aligned}
$$

where we set $W_{i+\frac{1}{2}}=w_{i+1}-w_{i}$. We use (3.1) and (3.2) to deal with the various terms in (4.5). Based on Taylor's expansions (3.3), we observe that

$$
w_{i+1}-w_{i}=\frac{1}{h} \int_{C_{i}}[w(x+h)-w(x)] d x=\frac{1}{h} \int_{C_{i}} \int_{0}^{h} w^{\prime}(x+s) d s d x ;
$$

therefore the following estimate holds:

$$
\left|W_{i+\frac{1}{2}}\right| \leq\left\|w^{\prime}\right\|_{L^{1}\left(C_{i}\right)}, \quad i \in \mathbb{Z} .
$$


Besides, the simplest first order approximation reads

$$
\begin{aligned}
w_{i+1}^{-}-w_{i}^{+} & =\left(1-\frac{\kappa_{i}}{2}-\frac{\lambda_{i+1}}{2}\right) \int_{C_{i}} w^{\prime}(\xi(x)) d x \\
& -\frac{\lambda_{i}}{2} \int_{C_{i}} w^{\prime}(\eta(x)) d x-\frac{\kappa_{i+1}}{2} \int_{C_{i}} w^{\prime}(\vartheta(x)) d x,
\end{aligned}
$$

for some $\xi(x), \eta(x), \vartheta(x) \in C_{i}$, so it follows from (4.6) that

$$
\left|w_{i+1}^{-}-w_{i}^{+}\right| \leq D_{i+\frac{1}{2}}\|w\|_{W^{1,1}} \quad \text { or } \quad\left|w_{i+1}^{-}-w_{i}^{+}\right| \leq D_{i+\frac{1}{2}} h\left\|w^{\prime}\right\|_{L^{\infty}} .
$$

Recalling that $\kappa_{i}+\lambda_{i}=1, \forall i \in \mathbb{Z}$, we obtain the second order approximation

$$
\begin{aligned}
w_{i+1}^{-}-w_{i}^{+} & =\left(1-\frac{\kappa_{i}}{2}-\frac{\lambda_{i+1}}{2}\right) \int_{C_{i}} w^{\prime \prime}(\xi(x))\left(x-x_{i}\right) d x \\
& +\frac{\lambda_{i}}{2} \int_{C_{i}} w^{\prime \prime}(\eta(x))\left(x-x_{i}\right) d x-\frac{3}{2} \kappa_{i+1} \int_{C_{i}} w^{\prime \prime}(\vartheta(x))\left(x-x_{i}\right) d x,
\end{aligned}
$$

for some $\xi(x), \eta(x), \vartheta(x) \in C_{i}$, and then it follows that

$$
\left|w_{i+1}^{-}-w_{i}^{+}\right| \leq D_{i+\frac{1}{2}} h\|w\|_{W^{2,1}} \quad \text { or } \quad\left|w_{i+1}^{-}-w_{i}^{+}\right| \leq D_{i+\frac{1}{2}} h^{2}\left\|w^{\prime \prime}\right\|_{L^{\infty}} .
$$

Remark 4.1. We note that the constant $D_{i+\frac{1}{2}}$ in (4.8) and (4.9) satisfies the estimate $D_{i+\frac{1}{2}} \leq \max \left\{\left(1-\frac{\kappa_{i}}{2}-\frac{\lambda_{i+1}}{2}\right), \frac{\lambda_{i}}{2}, \frac{3}{4} \kappa_{i+1}\right\} \leq 1$, uniformly for $i \in \mathbb{Z}$. Moreover, for any set of values $\left(\kappa_{i}, \lambda_{i}\right)_{i \in \mathbb{Z}}$, the bounds on these quantities are always not degenerate.

Finally, some long but straightforward computations, involving the third order expansions in (3.3), lead to conclude that

$$
\begin{aligned}
w_{i+1}^{-}-w_{i}^{+}=( & \left.\lambda_{i+1}-\kappa_{i}\right) \frac{h^{2}}{2} w^{\prime \prime}\left(x_{i}\right) \\
& +\left(1-\frac{\kappa_{i}}{2}-\frac{\lambda_{i+1}}{2}\right) \int_{C_{i}} w^{\prime \prime \prime}(\xi(x))\left(x-x_{i}\right)^{2} d x \\
& -\frac{\lambda_{i}}{2} \int_{C_{i}} w^{\prime \prime \prime}(\eta(x))\left(x-x_{i}\right)^{2} d x \\
& -\frac{\kappa_{i+1}}{2} \int_{C_{i}} w^{\prime \prime \prime}(\vartheta(x))\left(x-x_{i}\right)^{2} d x,
\end{aligned}
$$

for some $\xi(x), \eta(x), \vartheta(x) \in C_{i}$.

Remark 4.2. According to the piecewise linear reconstruction (4.1), discrete interfacial jumps approximate the second derivative of the numerical functions, as can be deduced from (4.5).

4.1. Stability estimate. The following result corresponds to that presented in Section 3.1 and we adapt the proof of Lemma 3.1 in the case of the discrete source operator (2.13).

Lemma 4.3. For the assumptions of Theorem 2.4, together with (2.7), there exists a constant $C:=C\left(L_{B},\left\|z^{\prime}\right\|_{L^{\infty}},\left\|z^{\prime \prime}\right\|_{L^{\infty}}\right)$, independent of $h$, such that

$$
\left.\left|\int_{\mathbb{R}} \mathcal{S}\left(u^{h} ; v^{h}\right)\right| e\right|^{p-1} \operatorname{sgn}(e) d x \mid \leq C\|e\|_{L^{p}}^{p} .
$$


Proof. From (2.17), we deduce for (2.13) that

$$
\begin{aligned}
& \int_{\mathbb{R}} \mathcal{S}\left(u^{h} ; v^{h}\right)|e|^{p-1} \operatorname{sgn}(e) d x \\
&=\int_{\mathbb{R}}\left\{\sum_{i \in \mathbb{Z}} \frac{1}{h}\left[\mathcal{B}^{+}\left(u_{i-1}^{+}, u_{i}^{-}, \Delta z_{i-\frac{1}{2}}\right)+\mathcal{B}^{-}\left(u_{i}^{+}, u_{i+1}^{-}, \Delta z_{i+\frac{1}{2}}\right)\right] \mathbb{1}_{C_{i}}\right. \\
&\left.-\sum_{i \in \mathbb{Z}} \frac{1}{h}\left[\mathcal{B}^{+}\left(v_{i-1}^{+}, v_{i}^{-}, \Delta z_{i-\frac{1}{2}}\right)+\mathcal{B}^{-}\left(v_{i}^{+}, v_{i+1}^{-}, \Delta z_{i+\frac{1}{2}}\right)\right] \mathbb{1}_{C_{i}}\right\}|e|^{p-1} \operatorname{sgn}(e) d x \\
&+\int_{\mathbb{R}}\left\{\sum_{i \in \mathbb{Z}} z_{i}^{\prime} b\left(u_{i}\right) \mathbb{1}_{C_{i}}-\sum_{i \in \mathbb{Z}} z_{i}^{\prime} b\left(v_{i}\right) \mathbb{1}_{C_{i}}\right\}|e|^{p-1} \operatorname{sgn}(e) d x \\
&= \sum_{i \in \mathbb{Z}}\left[\mathcal{B}^{+}\left(u_{i}^{+}, u_{i+1}^{-}, \Delta z_{i+\frac{1}{2}}\right)-\mathcal{B}^{+}\left(v_{i}^{+}, v_{i+1}^{-}, \Delta z_{i+\frac{1}{2}}\right)\right] e_{i+1}^{p-1} \\
&+\sum_{i \in \mathbb{Z}}\left[\mathcal{B}^{-}\left(u_{i}^{+}, u_{i+1}^{-}, \Delta z_{i+\frac{1}{2}}\right)-\mathcal{B}^{-}\left(v_{i}^{+}, v_{i+1}^{-}, \Delta z_{i+\frac{1}{2}}\right)\right] e_{i}^{p-1} \\
&+\sum_{i \in \mathbb{Z}} z_{i}^{\prime}\left[b\left(u_{i}\right)-b\left(v_{i}\right)\right] h e_{i}^{p-1}:=S_{1}+S_{2}+S_{3},
\end{aligned}
$$

where we have set $e_{i}^{p-1}=\frac{1}{h} \int_{C_{i}}|e|^{p-1} \operatorname{sgn}(e) d x$. To estimate $S_{1}$ and $S_{2}$, we proceed as in (3.5)-(3.6). According to (4.3), (4.4) and the Lipschitz properties of the numerical derivative (4.2), a simple computation shows that

$$
\left|u_{i}^{+}-v_{i}^{+}\right| \leq C\left(\left|u_{i}-v_{i}\right|+\left|u_{i+1}-v_{i+1}\right|+\left|u_{i-1}-v_{i-1}\right|\right),
$$

and an analogous relation is satisfied by $\left|u_{i}^{-}-v_{i}^{-}\right|, i \in \mathbb{Z}$. Hence, we can establish similar estimates to (3.7) and (3.8) also for the second order method. On the other hand, a direct treatment of the last term in (4.12) yields

$$
S_{3} \leq L_{B} \sum_{i \in \mathbb{Z}}\left|z_{i}^{\prime}\right|\left|u_{i}-v_{i}\right| h\left|e_{i}^{p-1}\right| .
$$

We give some details about the estimates of the numerical derivative (4.3), in the particular case of (2.1), that we will use later on the proofs. By performing appropriate expansions, also recalling that $\kappa_{i}+\lambda_{i}=1, \forall i \in \mathbb{Z}$, we obtain

$$
\begin{aligned}
z_{i}^{\prime} & =\frac{\kappa_{i}}{h} \int_{C_{i}} z^{\prime}(\xi(x)) d x+\frac{\lambda_{i}}{h} \int_{C_{i}} z^{\prime}(\eta(x)) d x \\
& =z^{\prime}\left(x_{i}\right)+\left(\kappa_{i}-\lambda_{i}\right) \frac{h}{2} z^{\prime \prime}\left(x_{i}\right)+\frac{\kappa_{i}}{3} h \int_{C_{i}} z^{\prime \prime \prime}(\vartheta(x)) d x+\frac{\lambda_{i}}{3} h \int_{C_{i}} z^{\prime \prime \prime}(\varrho(x)) d x,
\end{aligned}
$$

for some $\xi(x), \eta(x), \vartheta(x), \varrho(x) \in C_{i}$, which implies that $\left|z_{i}^{\prime}\right| \leq\left\|z^{\prime}\right\|_{L^{\infty}}$ in (4.13). For the arguments used in (3.9) and the first order approximation (4.8) of the values (2.12), we then conclude (4.11).

4.2. Consistency estimate. The proof of the following result is also an extension of that of Lemma 3.2 . 
Lemma 4.4. For the assumptions of Theorem 2.4 together with (2.7)-(2.9), there exists a constant $C:=C\left(L_{B}, K_{B},\left\|z^{\prime}\right\|_{L^{\infty}},\left\|z^{\prime \prime}\right\|_{L^{\infty}}\right)$, independent of $h$, such that

$$
\left.\left|\int_{\mathbb{R}} \mathcal{C}\left(u ; u^{h}\right)\right| e\right|^{p-1} \operatorname{sgn}(e) d x \mid \leq C h^{2}\left(\|z\|_{W^{3, p}}+\|u\|_{W^{2, p}}\right)\|e\|_{L^{p}}^{p-1} .
$$

Proof. We compute the integral of the discrete source operator (2.13), applied to the approximation (2.2) of the analytical solution,

$$
\begin{aligned}
& \int_{\mathbb{R}} B^{h}\left(x, u^{h}\right)|e|^{p-1} \operatorname{sgn}(e) d x \\
= & \sum_{i \in \mathbb{Z}}\left[\mathcal{B}^{+}\left(u_{i-1}^{+}, u_{i}^{-}, \Delta z_{i-\frac{1}{2}}\right)+\mathcal{B}^{-}\left(u_{i}^{+}, u_{i+1}^{-}, \Delta z_{i+\frac{1}{2}}\right)\right] e_{i}^{p-1}+\sum_{i \in \mathbb{Z}} z_{i}^{\prime} b\left(u_{i}\right) h e_{i}^{p-1},
\end{aligned}
$$

with $e_{i}^{p-1}=\frac{1}{h} \int_{C_{i}}|e|^{p-1} \operatorname{sgn}(e) d x$. Setting $\mathcal{B}=\mathcal{B}^{+}+\mathcal{B}^{-}$, we decompose the first part of the above formula into two terms, which we shall treat separately, namely

$$
T_{1}=\sum_{i \in \mathbb{Z}} \mathcal{B}\left(u_{i}^{+}, u_{i}^{+}, \Delta z_{i+\frac{1}{2}}\right) e_{i}^{p-1}
$$

and its remainder, which can be rewritten as

$$
\begin{aligned}
T_{2} & =\sum_{i \in \mathbb{Z}}\left[\mathcal{B}\left(u_{i}^{+}, u_{i+1}^{-}, \Delta z_{i+\frac{1}{2}}\right)-\mathcal{B}\left(u_{i}^{+}, u_{i}^{+}, \Delta z_{i+\frac{1}{2}}\right)\right] e_{i}^{p-1} \\
& +\sum_{i \in \mathbb{Z}}\left[\mathcal{B}^{+}\left(u_{i-1}^{+}, u_{i}^{-}, \Delta z_{i-\frac{1}{2}}\right)-\mathcal{B}^{+}\left(u_{i}^{+}, u_{i}^{-}, \Delta z_{i-\frac{1}{2}}\right)\right] e_{i}^{p-1} \\
& +\sum_{i \in \mathbb{Z}}\left[\mathcal{B}^{+}\left(u_{i}^{+}, u_{i}^{-}, \Delta z_{i-\frac{1}{2}}\right)-\mathcal{B}^{+}\left(u_{i}^{+}, u_{i}^{-}, \Delta z_{i+\frac{1}{2}}\right)\right] e_{i}^{p-1} \\
& +\sum_{i \in \mathbb{Z}}\left[\mathcal{B}^{+}\left(u_{i}^{+}, u_{i}^{-}, \Delta z_{i+\frac{1}{2}}\right)-\mathcal{B}^{+}\left(u_{i}^{+}, u_{i+1}^{-}, \Delta z_{i+\frac{1}{2}}\right)\right] e_{i}^{p-1} .
\end{aligned}
$$

We adopt the same procedure as in (3.12), by means of (2.7), to deduce

$$
\begin{aligned}
T_{2} \leq L_{B} \sum_{i \in \mathbb{Z}} & \left(\left|\Delta z_{i+\frac{1}{2}}\right|\left|u_{i+1}^{-}-u_{i}^{+}\right|+\left|\Delta z_{i-\frac{1}{2}}\right|\left|u_{i-1}^{+}-u_{i}^{+}\right|\right. \\
& \left.+\left|\Delta z_{i-\frac{1}{2}}-\Delta z_{i+\frac{1}{2}}\right|+\left|\Delta z_{i+\frac{1}{2}}\right|\left|u_{i}^{-}-u_{i+1}^{-}\right|\right)\left|e_{i}^{p-1}\right| .
\end{aligned}
$$

According to (4.3) and (4.4), we easily obtain

$$
\left|u_{i}^{+}-u_{i-1}^{+}\right|=\left|u_{i}-u_{i-1}+\frac{h}{2}\left(u_{i}^{\prime}-u_{i-1}^{\prime}\right)\right| \leq \int_{C_{i}}\left|u^{\prime}\right| d x,
$$

and an analogous estimate also holds for $\left|u_{i+1}^{-}-u_{i}^{-}\right|, i \in \mathbb{Z}$, while a second order approximation is needed for the central term in (4.17), that is,

$$
\left|\Delta z_{i+\frac{1}{2}}-\Delta z_{i-\frac{1}{2}}\right|=\left|\left(z_{i+1}-2 z_{i}+z_{i-1}\right)-\frac{h}{2}\left(z_{i+1}^{\prime}-z_{i-1}^{\prime}\right)\right| \leq h^{2} \int_{C_{i}}\left|z^{\prime \prime \prime}\right| d x .
$$


These estimates, together with (4.7) and (4.9), lead to us conclude that

$$
\begin{aligned}
T_{2} & \leq 3 L_{B} h\left\|z^{\prime \prime}\right\|_{L^{\infty}} \sum_{i \in \mathbb{Z}}\left(\int_{C_{i}}\left|u^{\prime}\right| d x\right)\left(\int_{C_{i}}|e|^{p-1} d x\right) \\
& +L_{B} h \sum_{i \in \mathbb{Z}}\left(\int_{C_{i}}\left|z^{\prime \prime \prime}\right| d x\right)\left(\int_{C_{i}}|e|^{p-1} d x\right) .
\end{aligned}
$$

Because of (4.10), the principal term (4.16) is further decomposed as

$$
\begin{aligned}
T_{1} & =\sum_{i \in \mathbb{Z}} \frac{\mathcal{B}\left(u_{i}^{+}, u_{i}^{+}, \Delta z_{i+\frac{1}{2}}\right)}{\Delta z_{i+\frac{1}{2}}}\left[\frac{\Delta z_{i+\frac{1}{2}}}{h}-Q_{i+\frac{1}{2}} \frac{h}{2} z^{\prime \prime}\left(x_{i}\right)\right] h e_{i}^{p-1} \\
& +\sum_{i \in \mathbb{Z}}\left[\frac{\mathcal{B}\left(u_{i}^{+}, u_{i}^{+}, \Delta z_{i+\frac{1}{2}}\right)}{\Delta z_{i+\frac{1}{2}}}-b\left(u_{i}^{+}\right)\right] Q_{i+\frac{1}{2}} \frac{h}{2} z^{\prime \prime}\left(x_{i}\right) h e_{i}^{p-1} \\
& +\sum_{i \in \mathbb{Z}} Q_{i+\frac{1}{2}} \frac{h}{2} z^{\prime \prime}\left(x_{i}\right) b\left(u_{i}^{+}\right) h e_{i}^{p-1}:=T_{1}^{1}+T_{1}^{2}+T_{1}^{3},
\end{aligned}
$$

where we set $Q_{i+\frac{1}{2}}=\lambda_{i+1}-\kappa_{i} \leq 1, \forall i \in \mathbb{Z}$. We give a few details of the estimate for each part. We proceed as in (3.16) and we obtain from (4.10) that

$$
T_{1}^{1} \leq L_{B} h \sum_{i \in \mathbb{Z}}\left(\int_{C_{i}}\left|z^{\prime \prime \prime}\right| d x\right)\left(\int_{C_{i}}|e|^{p-1} d x\right) .
$$

Besides, using (4.7), we derive from the consistency condition (2.9) that

$$
T_{1}^{2} \leq K_{B} h\left\|z^{\prime \prime}\right\|_{L^{\infty}} \sum_{i \in \mathbb{Z}}\left(\int_{C_{i}}\left|z^{\prime}\right| d x\right)\left(\int_{C_{i}}|e|^{p-1} d x\right) .
$$

We then pass to the crucial point of the proof, to show the convergence towards the integral of the analytical source operator (1.3). On the one hand, by applying classical Taylor expansions, we have

$$
\begin{aligned}
\int_{\mathbb{R}} B(x, u)|e|^{p-1} \operatorname{sgn}(e) d x & =\sum_{i \in \mathbb{Z}} \int_{C_{i}} z^{\prime}\left(x_{i}\right) b\left(u\left(x_{i}\right)\right)|e|^{p-1} \operatorname{sgn}(e) d x \\
& +\sum_{i \in \mathbb{Z}} \int_{C_{i}}\left(z^{\prime} b(u)\right)^{\prime}\left(\xi\left(x_{i}\right)\right)\left(x-x_{i}\right)|e|^{p-1} \operatorname{sgn}(e) d x,
\end{aligned}
$$

for some $\xi\left(x_{i}\right) \in C_{i}$. On the other hand, recalling the definition of interfacial values (2.11) - 2.12) and for the regularity assumed in (1.3), we can write

$$
b\left(u_{i}^{+}\right)=b\left(u_{i}\right)+b^{\prime}\left(\nu_{i}\right) \frac{h}{2} u_{i}^{\prime}, \quad\left|b^{\prime}\left(\nu_{i}\right)\right| \leq L_{B}, \forall i \in \mathbb{Z},
$$

so that from (4.19) we deduce

$$
T_{1}^{3}=\sum_{i \in \mathbb{Z}} Q_{i+\frac{1}{2}} \frac{h}{2} z^{\prime \prime}\left(x_{i}\right) b\left(u_{i}\right) h e_{i}^{p-1}+R_{1}
$$

and we use analogous approximations to 4.14 for the numerical derivatives of the analytical solution to obtain

$$
R_{1} \leq L_{B} h\left\|z^{\prime \prime}\right\|_{L^{\infty}} \sum_{i \in \mathbb{Z}}\left(\int_{C_{i}}\left|u^{\prime}\right| d x\right)\left(\int_{C_{i}}|e|^{p-1} d x\right) .
$$


To conclude, we take into account the contribution of the additional term in the discrete source operator (2.13). The following second order approximation of cellaverages on the mesh,

$$
u_{i}=u\left(x_{i}\right)+R_{i}, \quad R_{i}=\frac{1}{h} \int_{C_{i}} u^{\prime \prime}(\xi(x)) \frac{\left(x-x_{i}\right)^{2}}{2} d x
$$

for some $\xi(x) \in C_{i}$, associated with the standard Taylor expansion

$$
b\left(u_{i}\right)=b\left(u\left(x_{i}\right)\right)+b^{\prime}\left(\nu_{i}\right) R_{i}, \quad\left|b^{\prime}\left(\nu_{i}\right)\right| \leq L_{B}, \forall i \in \mathbb{Z},
$$

and proceeding according to (4.14), leads to

$$
\begin{aligned}
\sum_{i \in \mathbb{Z}} z_{i}^{\prime} b\left(u_{i}\right) h e_{i}^{p-1} & =\sum_{i \in \mathbb{Z}} z^{\prime}\left(x_{i}\right) b\left(u\left(x_{i}\right)\right) h e_{i}^{p-1}+R_{2} \\
& +\sum_{i \in \mathbb{Z}} P_{i+\frac{1}{2}} \frac{h}{2} z^{\prime \prime}\left(x_{i}\right) b\left(u_{i}\right) h e_{i}^{p-1}+R_{3},
\end{aligned}
$$

where we set $P_{i+\frac{1}{2}}=\kappa_{i}-\lambda_{i}, i \in \mathbb{Z}$, while the remainders are estimated as

$$
\begin{aligned}
& R_{2} \leq L_{B} h\left\|z^{\prime}\right\|_{L^{\infty}} \sum_{i \in \mathbb{Z}}\left(\int_{C_{i}}\left|u^{\prime \prime}\right| d x\right)\left(\int_{C_{i}}|e|^{p-1} d x\right), \\
& R_{3} \leq L_{B} h \sum_{i \in \mathbb{Z}}\left(\int_{C_{i}}\left|z^{\prime \prime \prime}\right| d x\right)\left(\int_{C_{i}}|e|^{p-1} d x\right) .
\end{aligned}
$$

Therefore, up to bounded remainders (4.24), (4.28) and (4.29), by combining (4.22), (4.23) and (4.27), we have

$$
\begin{aligned}
\int_{\mathbb{R}} B(x, u)|e|^{p-1} \operatorname{sgn}(e) d x-T_{1}^{3}-\sum_{i \in \mathbb{Z}} z_{i}^{\prime} b\left(u_{i}\right) h e_{i}^{p-1} \\
=\sum_{i \in \mathbb{Z}} \int_{C_{i}} z^{\prime \prime}\left(\xi\left(x_{i}\right)\right) b\left(u\left(\xi\left(x_{i}\right)\right)\right)\left(x-x_{i}\right)|e|^{p-1} \operatorname{sgn}(e) d x \\
\quad+\sum_{i \in \mathbb{Z}} \int_{C_{i}} z^{\prime}\left(\xi\left(x_{i}\right)\right) b^{\prime}\left(u\left(\xi\left(x_{i}\right)\right)\right) u^{\prime}\left(\xi\left(x_{i}\right)\right)\left(x-x_{i}\right)|e|^{p-1} \operatorname{sgn}(e) d x \\
\quad-\sum_{i \in \mathbb{Z}}\left(P_{i+\frac{1}{2}}+Q_{i+\frac{1}{2}}\right) \frac{h}{2} z^{\prime \prime}\left(x_{i}\right) b\left(u\left(x_{i}\right)\right) h e_{i}^{p-1} \\
-\sum_{i \in \mathbb{Z}}\left(P_{i+\frac{1}{2}}+Q_{i+\frac{1}{2}}\right) \frac{h}{2} z^{\prime \prime}\left(x_{i}\right) b^{\prime}\left(\nu_{i}\right) R_{i} h e_{i}^{p-1},
\end{aligned}
$$

where again we used (4.25) - (4.26) and $P_{i+\frac{1}{2}}+Q_{i+\frac{1}{2}}=\lambda_{i+1}-\lambda_{i}, i \in \mathbb{Z}$. We introduce an appropriate hypothesis on the slope limiter in (4.2), as discussed in Section 2.2 namely an additional condition for its coefficients (4.3), that is,

$$
\exists \Lambda_{0}>0 \text { such that } \lambda_{i+1}-\lambda_{i} \geq \Lambda_{0}, \forall i \in \mathbb{Z} .
$$


This condition allows us to rewrite the difference between the first and third terms in the right-hand side of (4.30) in an integral form to derive

$$
\begin{aligned}
\left.\left|\sum_{i \in \mathbb{Z}} \int_{C_{i}} z^{\prime \prime}\left(\xi\left(x_{i}\right)\right) b\left(u\left(\xi\left(x_{i}\right)\right)\right)\left(x-x_{i}\right)\right| e\right|^{p-1} \operatorname{sgn}(e) d x \\
\quad-\sum_{i \in \mathbb{Z}}\left(P_{i+\frac{1}{2}}+Q_{i+\frac{1}{2}}\right) \frac{h}{2} z^{\prime \prime}\left(x_{i}\right) b\left(u\left(x_{i}\right)\right) h e_{i}^{p-1} \mid \\
\leq h \sum_{i \in \mathbb{Z}}\left(\int_{C_{i}}\left|\left(z^{\prime \prime} b(u)\right)^{\prime}\right| d x\right)\left(\int_{C_{i}}|e|^{p-1} d x\right) \\
\leq L_{B} h \sum_{i \in \mathbb{Z}}\left(\int_{C_{i}}\left|z^{\prime \prime \prime}\right| d x\right)\left(\int_{C_{i}}|e|^{p-1} d x\right) \\
\quad+L_{B} h\left\|z^{\prime \prime}\right\|_{L^{\infty}} \sum_{i \in \mathbb{Z}}\left(\int_{C_{i}}\left|u^{\prime}\right| d x\right)\left(\int_{C_{i}}|e|^{p-1} d x\right) .
\end{aligned}
$$

Similarly, we can prove an estimate for the difference between the second and fourth terms in the right-hand side of (4.30), which is analogous to (4.28). We apply the same arguments as in Lemma 3.2 to estimate (4.18), (4.20), (4.21) and (4.32), with its related computations, to conclude the proof of 4.15).

4.3. Proof of Theorem $\mathbf{2 . 4}$ and Theorem 2.5 . We proceed as in Section 3.3 by using the stability estimate (4.11) and the consistency estimate (4.15), to prove the second order error estimate (2.20).

The proof of Theorem [2.5] involves the main tools introduced for the results in Section 3 and Section 4. Because of the consistency hypotheses (2.7)-(2.8) and (2.15), the same techniques as in Lemma 3.2 extend to the case of the discrete source operator (2.14), while we can apply the arguments formulated in Lemma 4.3 to deduce analogous stability estimates.

\section{REMARKS AND NUMERICAL EVIDENCE}

The principal issue in the proof of Theorem 2.3 and Theorem 2.4 is to establish consistency estimates (3.10) and (4.15), in particular to show the convergence of the numerical source operator towards the analytical source term (1.3) from the relation (3.18) and (4.23), respectively.

TABLE 1. VanLeer limiter with TVD reconstruction

\begin{tabular}{|c|c|c|c|c|c|c|}
\hline & \multicolumn{2}{|c|}{$\|e(t)\|_{L^{1}}$} & \multicolumn{2}{c|}{$\|e(t)\|_{L^{2}}$} & \multicolumn{2}{c|}{$\|e(t)\|_{L^{\infty}}$} \\
\hline & Error & Rate & Error & Rate & Error & Rate \\
\hline 50 & $0.323743 \mathrm{E}-02$ & & $0.772868 \mathrm{E}-02$ & & $0.368811 \mathrm{E}-01$ & \\
\hline 100 & $0.816610 \mathrm{E}-03$ & 1.987 & $0.270992 \mathrm{E}-02$ & 1.512 & $0.184893 \mathrm{E}-01$ & 0.996 \\
\hline 200 & $0.207343 \mathrm{E}-03$ & 1.982 & $0.951254 \mathrm{E}-03$ & 1.511 & $0.921217 \mathrm{E}-02$ & 1.001 \\
\hline 400 & $0.516765 \mathrm{E}-04$ & 1.990 & $0.336009 \mathrm{E}-03$ & 1.508 & $0.461648 \mathrm{E}-02$ & 0.999 \\
\hline 800 & $0.128919 \mathrm{E}-04$ & 1.993 & $0.118745 \mathrm{E}-03$ & 1.506 & $0.231075 \mathrm{E}-02$ & 0.999 \\
\hline 1600 & $0.321149 \mathrm{E}-05$ & 1.995 & $0.419888 \mathrm{E}-04$ & 1.505 & $0.115659 \mathrm{E}-02$ & 0.999 \\
\hline
\end{tabular}


TABLE 2. VanLeer limiter with ENO reconstruction

\begin{tabular}{|c|c|c|c|c|c|c|}
\hline & \multicolumn{2}{|c|}{$\|e(t)\|_{L^{1}}$} & \multicolumn{2}{c|}{$\|e(t)\|_{L^{2}}$} & \multicolumn{2}{c|}{$\|e(t)\|_{L^{\infty}}$} \\
\hline & Error & Rate & Error & Rate & Error & Rate \\
\hline 50 & $0.129563 \mathrm{E}-02$ & & $0.148671 \mathrm{E}-02$ & & $0.324608 \mathrm{E}-02$ & \\
\hline 100 & $0.326417 \mathrm{E}-03$ & 1.989 & $0.368537 \mathrm{E}-03$ & 2.012 & $0.814699 \mathrm{E}-03$ & 1.994 \\
\hline 200 & $0.819300 \mathrm{E}-04$ & 1.992 & $0.917495 \mathrm{E}-04$ & 2.009 & $0.203996 \mathrm{E}-03$ & 1.996 \\
\hline 400 & $0.205217 \mathrm{E}-04$ & 1.993 & $0.228873 \mathrm{E}-04$ & 2.007 & $0.509962 \mathrm{E}-04$ & 1.997 \\
\hline 800 & $0.513540 \mathrm{E}-05$ & 1.995 & $0.571567 \mathrm{E}-05$ & 2.006 & $0.127479 \mathrm{E}-04$ & 1.998 \\
\hline 1600 & $0.128447 \mathrm{E}-05$ & 1.996 & $0.142815 \mathrm{E}-05$ & 2.005 & $0.318655 \mathrm{E}-05$ & 1.998 \\
\hline
\end{tabular}

We point out that, due to the introduction of piecewise linear reconstructions of the function $z$, the differences of discrete interfacial values approximate the second order derivative, as given in (4.10), and the upwind part (4.23) of the discretization (2.13) "overtakes" the desired result; an additional term (4.27) is thus needed to recover the first order derivatives from the Taylor expansions of the source term (4.22). Moreover, some restrictions (4.31) on the definition of the slope limiter are also required, to guarantee the occurrence of suitable error estimates (refer also to [5, [21] and 22]). Without these assumptions, only suboptimal results are derived (see [18] and [34], for instance).

These questions can also be justified numerically. Tables 1 and 2 reproduce the convergence rates for the U.S.I. method applied to the simple problem

$$
\partial_{t} u=z^{\prime}(x), \quad u(0, x)=u_{0}(x),
$$

with $z(x)=\sin (\pi x), x \in[0,1]$, for which an analytical solution is available to make direct comparisons, i.e., $u(t, x)=u_{0}(x)+z^{\prime}(x) t$. The results correspond to the discretization (2.13) for the standard VanLeer limiter 32, associated with a simple TVD reconstruction (see [31]) in Table 1 and with an appropriate ENO reconstruction (see 13 ) in Table 2.

The problems just discussed do not generally arise in the case of the second order discretization (2.14), for which stronger consistency hypotheses (2.15) are made, to compensate for reduced accuracy in the reconstructions.

Although the question of preserving stationary solutions at the discrete level is only handled rigorously for first order U.S.I. methods (see [29] and its references), the numerical results obtained for the Saint-Venant system indicate that the second order discretization (2.13) exactly simulates simple steady states (refer to [16] for details). As far as we know, similar issues are only addressed in [4] and [9]. Further applications of these methods to different situations are also proposed in [2].

\section{REFERENCES}

1. V.B. Barakhnin, TVD scheme of second-order approximation on a nonstationary adaptive grid for hyperbolic systems, Russian J. Numer. Anal. Math. Modelling, 16 (2001), no. 1, 1-17. MR 2002g:65093

2. M. Ben-Artzi, J. Falcovitz, An upwind second-order scheme for compressible duct flows, SIAM J. Sci. Statist. Comput., 7 (1986), no. 3, 744-768. MR 88a:65107]

3. C. Chainais-Hillairet, S. Champier, Finite volume schemes for nonhomogeneous scalar conservation laws: error estimate, Numer. Math., 88 (2001), no. 4, 607-639. MR 2002b:65150

4. A. Chalabi, Stable upwind schemes for hyperbolic conservation laws with source terms, IMA J. Numer. Anal., 12 (1992), no. 2, 217-241. MR 93c:65108 
5. A. Chalabi, On convergence of numerical schemes for hyperbolic conservation laws with stiff source terms, Math. Comp., 66 (1997), no. 218, 527-545. MR 97g:65178

6. M.G. Crandall, A. Majda, Monotone difference approximations for scalar conservation laws, Math. Comp., 34 (1980), no. 149, 1-21. MR 81b:65079

7. C.M. Dafermos, Hyperbolic conservation laws in continuum physics, Grundlehren der Mathematischen Wissenschaften (Fundamental Principles of Mathematical Sciences) 325, SpringerVerlag, Berlin, 2000. MR 2001m:35212

8. C.M. Dafermos, L. Hsiao, Hyperbolic systems and balance laws with inhomogeneity and dissipation, Indiana Univ. Math. J., 31 (1982), no. 4, 471-491. MR 83m:35093

9. L. Gascón, J.M. Corberán, Construction of second-order TVD schemes for nonhomogeneous hyperbolic conservation laws, J. Comput. Phys., 172 (2001), no. 1, 261-297. MR 2002h:65125

10. E. Godlewski, P.A. Raviart, Hyperbolic systems of conservation laws, Mathématiques \& Applications 3/4, Ellipses, Paris, 1991. MR 95i:65146

11. L. Gosse, Sur la stabilité des approximations implicites des lois de conservation scalaires non homogènes, C. R. Acad. Sci. Paris Sér. I Math., 329 (1999), no. 1, 79-84. MR 2000d:65152

12. L. Gosse, Localization effects and measure source terms in numerical schemes for balance laws, Math. Comp., 71 (2002), no. 238, 553-582. MR 2003e:65147

13. A. Harten, S. Osher, Uniformly high-order accurate nonoscillatory schemes I, SIAM J. Numer. Anal., 24 (1987), no. 2, 279-309. MR 90a:65198

14. A. Harten, B. Engquist, S. Osher, S.R. Chakravarthy, Uniformly high-order accurate essentially nonoscillatory schemes III, J. Comput. Phys., 71 (1987), no. 2, 231-303. MR 90a:65199

15. M.E. Hubbard, Multidimensional slope limiters for MUSCL-type finite volume schemes on unstructured grids, J. Comput. Phys., 155 (1999), no. 1, 54-74. MR 2000f:76081

16. Th. Katsaounis, C. Simeoni, Second order approximation of the viscous Saint-Venant system and comparison with experiments, Hyperbolic Problems: Theory, Numerics, Applications (T. Hou and E. Tadmor, Eds.), Springer, 2003.

17. S.N. Kružkov, First order quasilinear equations with several independent variables, Math. Sb. (N.S.), 81 (1970), n. 123, 228-255. MR 42:2159

18. A.Y. LeRoux, Convergence of an accurate scheme for first order quasilinear equations, RAIRO Anal. Numér., 15 (1981), no. 2, 151-170. MR 83g:65089

19. A.Y. LeRoux, M.N. LeRoux, Convergence d'un schéma à profils stationnaires pour les équations quasi linéaires du premier ordre avec termes sources, C. R. Acad. Sci. Paris Sér. I Math., 333 (2001), no. 7, 703-706. MR 2003a:65066

20. R.J. LeVeque, Numerical methods for conservation laws, Lectures in Mathematics ETH Zürich, Birkhäuser Verlag, Basel, 1990. MR 91j:65142

21. D. Levy, G. Puppo, G. Russo, Compact central WENO schemes for multidimensional conservation laws, SIAM J. Sci. Comput., 22 (2000), no. 2, 656-672. MR 2001d:65110

22. D. Levy, G. Puppo, G. Russo, Central WENO schemes for hyperbolic systems of conservation laws, M2AN Math. Model. Numer. Anal., 33 (1999), no. 3, 547-571. MR 2000f:65079

23. M. Louaked, L. Hanich, Un schéma TVD-multirésolution pour les équations de Saint-Venant, C. R. Acad. Sci. Paris Sér. I Math., 331 (2000), no. 9, 745-750. MR 2001j:76079

24. H. Nessyahu, E. Tadmor, Nonoscillatory central differencing for hyperbolic conservation laws, J. Comput. Phys., 87 (1990), no. 2, 408-463. MR 91i:65157

25. S. Osher, P.K. Sweby, Recent developments in the numerical solution of nonlinear conservation laws, The state of the art in numerical analysis (Birmingham, 1986), Inst. Math. Appl. Conf. Ser. New Ser., 9, Oxford Univ. Press, New York, 1987, 681-701. MR 88j:65177

26. S. Osher, E. Tadmor, On the convergence of difference approximations to scalar conservation laws, Math. Comp., 50 (1988), no. 181, 19-51. MR 89m:65086

27. P. de Oliveira, J. Santos, A converging finite volume scheme for hyperbolic conservation laws with source terms, Numerical methods for differential equations (Coimbra, 1998), J. Comput. Appl. Math., 111 (1999), no. 1-2, 239-251. MR 2001g:65129

28. P. de Oliveira, J. Santos, On a class of high resolution methods for solving hyperbolic conservation laws with source terms, Applied nonlinear analysis, Kluwer/Plenum, New York, 1999, 403-416. MR 2000k:65152

29. B. Perthame, C. Simeoni, Convergence of the Upwind Interface Source method for hyperbolic conservation laws, Hyperbolic Problems: Theory, Numerics, Applications (T. Hou and E. Tadmor, Eds.), Springer, 2003. 
30. C.-W. Shu, Essentially non-oscillatory and weighted essentially non-oscillatory schemes for hyperbolic conservation laws, Advanced numerical approximation of nonlinear hyperbolic equations (Cetraro, 1997), Lecture Notes in Math., 1697, Springer, Berlin, 1998, 325-432. MR 2001a:65096

31. P.K. Sweby, TVD schemes for inhomogeneous conservation laws, Nonlinear hyperbolic equations: theory, computation methods and applications (Aachen, 1988), Notes Numer. Fluid Mech., 24, Vieweg, Braunschweig, 1989, 599-607.

32. B. VanLeer, Towards the ultimate conservative difference scheme $V$. A second-order sequel to Godunov's method, J. Comput. Phys., 32 (1979), no. 1, 101-136.

33. A. Vasseur, Well-posedness of scalar conservation laws with singular sources, Methods Appl. Anal. 9 (2002), no. 2, 291-312.

34. J.P. Vila, Systèmes de lois de conservation, schémas quasi d'ordre 2 et condition d'entropie, C. R. Acad. Sci. Paris Sér. I Math., 299 (1984), n. 5, 157-160. MR 85h:65202

35. J.P. Vila, An analysis of a class of second-order accurate Godunov-type schemes, SIAM J. Numer. Anal., 26 (1989), no. 4, 830-853. MR 90g:65120

Department of Applied Mathematics, University of Crete, GR 71409 Heraklion, Crete, Greece; I.A.C.M.-F.O.R.T.H., GR 71110 Heraklion, Crete, Greece

E-mail address: thodoros@tem.uoc.gr

Département de Mathématiques et Applications, École Normale Supérieure, 45, Rue D'Ulm, 75230 Paris Cedex 05, France; I.A.C.M.-F.O.R.T.H., GR 71110 Heraklion, Crete, GREECE

E-mail address: simeoni@dma.ens.fr, simeoni@tem.uoc.gr 